\begin{tabular}{|c|l|}
\hline Title & $\begin{array}{l}\text { Identification of residues important for the catalysis, structure maintenance, and substrate specificity of yeast 3 } \\
\text { hydroxyacyl-CoA dehydratase Phs1 }\end{array}$ \\
\hline Author(s) & Yazawa, Tomoyo; Naganuma, Tatsuro; Y amagata, Maki; Kinara, A kio \\
\hline Citation & $\begin{array}{l}\text { FEBS LETTERS, 587(6), 804.809 } \\
\text { https://doi.org/10.1016).febslet.2013.02.006 }\end{array}$ \\
\hline Issue Date & 2013-03-18 \\
\hline Doc URL & http://hdl.handle.net/2115/52639 \\
\hline Type & article (author version) \\
\hline Additional Information & There are other files related to this item in HUSCAP. Check the above URL. \\
\hline File Information & ManuscriptandFig.pdf \\
\hline
\end{tabular}

Instructions for use 


\title{
Identification of residues important for the catalysis, structure maintenance, and substrate specificity of yeast 3-hydroxyacyl-CoA dehydratase Phs1
}

Tomoyo Yazawa, Tatsuro Naganuma, Maki Yamagata, and Akio Kihara*

Laboratory of Biochemistry, Faculty of Pharmaceutical Sciences, Hokkaido University, Japan

*Corresponding author

Address correspondence to:

\author{
Professor Akio Kihara \\ Laboratory of Biochemistry \\ Faculty of Pharmaceutical Sciences, Hokkaido University \\ Kita 12-jo, Nishi 6-chome, Kita-ku, Sapporo 060-0812, Japan \\ Telephone: +81-11-706-3754 \\ Fax: +81-11-706-4900 \\ E-mail: kihara@pharm.hokudai.ac.jp
}

Keywords: Very long-chain fatty acid; 3-Hydroxyacyl-CoA dehydratase; Fatty acid; Lipid; Phs1; Membrane 


\begin{abstract}
Yeast Phs1 is a 3-hydroxyacyl-CoA dehydratase involved in the very long-chain fatty acid elongation. In the present study, we biochemically characterized Phs1 mutants with Ala-substitution at each of seven highly conserved amino-acid residues. All mutants exhibited reduced Phs1 activity. The E60A, Q79A, and R141A mutants were sensitive to digitonin, indicative of their reduced structural integrity. The fatty acid elongation cycle was greatly inhibited in the R83A, R141A, and G152A mutant membranes. The enzyme kinetics study implicated the direct involvement of the Arg83 and Gly152 residues in the catalytic process. The E60A mutation was found to affect the substrate specificity.
\end{abstract}

Abbreviations: VLCFA, very long-chain fatty acid; FA, fatty acid; LCFA, long-chain fatty acid; SC, synthetic complete; FAME, fatty acid methyl ester. 


\section{Introduction}

Very long-chain fatty acids (VLCFAs) are fatty acids (FAs) with a chain-length greater than 20 carbon atoms (C20). Although much less abundant than long-chain FAs (LCFAs; C11-C20), VLCFAs play an important physiological function that cannot be substituted for by LCFAs, such as retinal function, spermatogenesis, skin barrier formation, and inflammation [1]. For example, VLCFA-deficient yeast as well as mice is known to be inviable [2,3]. VLCFAs are also pathologically important, and several inherited disorders have been linked to mutations in VLCFA-synthesizing and -degrading genes [1].

VLCFAs are synthesized on the endoplasmic reticulum membrane from LCFAs through the FA elongation cycle. In each cycle, an acyl-CoA substrate is converted to an acyl-CoA product with 2 additional carbons by 4 sequential reactions (condensation, reduction, dehydration, and reduction) (Fig. S1A). The rate-limiting step is the first reaction, involving condensation of an acyl-CoA substrate with malonyl-CoA as a C2 donor [4]. 3-Hydroxyacyl-CoA dehydratase is an enzyme that catalyzes the third reaction. In 2007, PHS1 was identified in yeast as the gene encoding 3-hydroxyacyl-CoA dehydratase [5]. Phs1 is highly conserved among eukaryotes, and mammals have four Phs1 homologues (HACD1-4) [6].

In yeast, VLCFAs are predominantly used for the synthesis of sphingolipids, which are one of the major lipid components of the eukaryotic plasma membrane with many biological functions [7]. The sphingolipid backbone (ceramide) is composed of a long-chain base and an amide-linked FA (C26 for yeast; mainly C16 and C24 for mammals) [1,7]. Therefore, any mutation in a gene involved in VLCFA production interferes with sphingolipid synthesis. For example, when the yeast PHS1 mRNA was not transcribed using a controllable tetracycline 
(doxycyclin)-dependent promoter (Tet-PHS1 cells), the complex sphingolipid level was decreased, whereas the ceramide and long-chain base levels were increased [8].

Phs1 is an integral membrane protein with six membrane-spanning domains [8]. However, Phs1 and its family members have no known domains or sequence motifs and exhibit no sequence similarity to other protein families. To identify the amino-acid residues important for Phs1 function, we previously created Phs1 mutants by substituting each of 22 amino-acid residues, which are conserved among 31 Phs1 family members, with Ala, and introduced each mutant into Tet-PHS1 cells [8]. The cells producing one of 9 mutant proteins (E60A, Q79A, R83A, E116A, R119A, R141A, Y149A, G152A, or E156A) accumulated the long-chain base phytosphingosine, indicative of a defect in Phs1 activity [8]. In addition, two of these 9 mutants (Y149A and E156A) neither complemented the growth defect of Tet-PHS1 cells in the presence of doxycyclin nor exhibited the enzyme activity. Thus, both Tyr149 and Glu156 residues were considered to be a part of the active site. Although the rest of the 7 amino-acid residues were expected to be important for Phs1 function, their precise role still remains unclear. In the present study, we have performed several biochemical analyses on the 7 remaining mutants and identified the amino-acid residues important for the catalysis, structural maintenance, and substrate specificity of Phs1. 


\section{Materials and methods}

\subsection{Yeast strains, plasmids, and media}

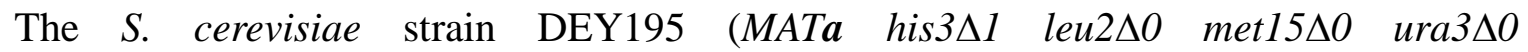
pep4A::MET25) is a derivative of BY4741 [9]. The plasmid encoding the wild-type PHS1-3xFLAG (pSH14) or the mutant PHS1-3xFLAG (pSH18, E60A; pSH19, Q79A; pSH20, R83A; pSH22, E116A; pSH23, R119A; pSH25, R141A; and pSH30, G152A) under the control of MET25 promoter was introduced into the DEY195 cells. The phs $1 \Delta:: L E U 2$ mutation was introduced into each of the resultant cells, generating YTY19 (wild type), YTY20 (E60A), YTY21 (Q79A), YTY22 (R83A), YTY23 (E116A), YTY24 (R119A), YTY25 (R141A), and YTY26 (G152A), respectively. All the plasmids were constructed as described previously [8]. Cells were grown in synthetic complete (SC) medium $(0.67 \%$ yeast nitrogen base, 2\% D-glucose, and nutritional supplements) but lacking histidine and methionine (SC-His-Met) at $30{ }^{\circ} \mathrm{C}$.

\subsection{In vivo 3-hydroxyacyl-CoA dehydratase assay}

Yeast spheroplasts prepared as described previously [10] were suspended in buffer A (50 mM HEPES-KOH (pH 6.8), $150 \mathrm{mM} \mathrm{KOAc,} 2 \mathrm{mM} \mathrm{Mg}(\mathrm{OAc})_{2}, 1 \mathrm{mM} \mathrm{CaCl}{ }_{2}, 200 \mathrm{mM}$ sorbitol, $1 \mathrm{mM}$ dithiothreitol, $1 \mathrm{mM}$ phenylmethylsulfonyl fluoride, and a $1 \mathrm{X}$ Complete ${ }^{\mathrm{TM}}$ protease inhibitor mixture (EDTA-free; Roche Diagnostics, Indianapolis, IN, USA) and lysed by sonication. Unlysed cells were removed by centrifugation at $2,000 \mathrm{x} \mathrm{g}$ for $3 \mathrm{~min}$. The supernatant was centrifuged at $100,000 \mathrm{x}$ g for $30 \mathrm{~min}$ at $4{ }^{\circ} \mathrm{C}$, and the resulting pellet (total membrane fractions) was suspended in buffer A. The assay was performed using 3-hydroxy $\left[1-{ }^{14} \mathrm{C}\right]$ palmitoyl-CoA $(55 \mathrm{mCi} / \mathrm{mmol}$; American Radiolabeled Chemicals, St. 
Louis, MO, USA) as described previously [8].

\subsection{In vitro FA elongase assay}

The assay was performed using acyl-CoAs (C16 to $\mathrm{C} 24)$ and $\left[2-{ }^{14} \mathrm{C}\right]$ malonyl-CoA (55 $\mathrm{mCi} / \mathrm{mmol}$; American Radiolabeled Chemicals) as described previously [11].

\subsection{Immunoblotting}

Immunoblotting was performed as described previously [8], using the anti-FLAG antibody M2 (1 $\mu \mathrm{g} / \mathrm{ml}$; Stratagene, Agilent Technologies, La Jolla, CA, USA) and anti-Pma1 (yN-20) antibody $(0.4 \mu \mathrm{g} / \mathrm{ml}$; Santa Cruz Biotechnology, Santa Cruz, CA, USA) as the primary antibodies, and HRP-conjugated anti-mouse and anti-goat $\operatorname{IgG~} \mathrm{F}\left(\mathrm{ab}{ }^{\prime}\right)_{2}$ fragments (each at 1:7500 dilution; GE Healthcare Bio-Sciences, Piscataway, NJ, USA) as the secondary antibodies. Labeling was detected using Pierce Western Blotting Substrate (Thermo Fisher Scientific, Waltham, MA, USA). 


\section{Results}

3.1. The Glu60, Gln79, Arg83, Glu116, Arg119, Arg141, and Gly152 residues are important for the enzyme activity of Phs1

We individually substituted the 7 conserved amino-acid residues of Phs1 (Glu60, Gln79, Arg83, Glu116, Arg119, Arg141, and Gly152) with Ala and constructed phs1s strains expressing each of these Ala-substituted mutants (E60A, Q79A, R83A, E116A, R119A, R141A, and G152A) as an N-terminally 3xFLAG-tagged protein. Using total membrane fractions prepared from these yeast cells and 3-hydroxy $\left[1-{ }^{14} \mathrm{C}\right]$ palmitoyl-CoA as a substrate, we examined their 3-hydroxyacyl-CoA dehydratase activity (Fig. 1A). All the mutants exhibited reduced activities compared to the wild-type Phs1, with R83A mutant having the lowest activity. Their intracellular level was similar or slightly higher compared to that of the wild-type Phs1, except for the E60A and R119A mutants whose levels were lower (Fig. 1B).

We then repeated the assay in the presence of the non-ionic detergent digitonin (Fig. 1C). The activity of the R83A, E116A, R119A, and G152A mutants, as well as the wild-type Phs1, was less affected by treatment with digitonin; however, the activity of the E60A, Q79A, and R141A mutants was significantly reduced (Fig. 1C). These results suggest that the mutation in the Glu60, Gln79, or Arg141 residue affect the structural integrity of Phs1.

3.2. The R83A, R141A, and G152A mutations cause a decrease in acyl-CoA production during the FA elongation cycle

FA elongation takes place by cycling through a 4-step reaction, and each step is catalyzed by a specific enzyme (Fig. S1A). To examine the effect of the PHS1 mutation on the overall FA elongation cycle, we performed an in vitro FA elongation assay using total membrane 
fractions, palmitoyl-CoA (C16-CoA) (the substrate of the first step) and $\left[{ }^{14} \mathrm{C}\right]$ malonyl-CoA. The reaction products were analyzed by normal-phase TLC after hydrolysis to the corresponding FA derivatives (Fig. 2A and B). Since the first condensation step is the rate-limiting step of the FA elongation cycle, acyl-CoA (the product; FA in the figure) was detected as the only product of the wild-type membrane. On the other hand, all the mutant membranes accumulated 3-hydroxyacyl-CoA (the substrate of Phs1) (3-hydroxy-FA in the figure) in addition to the acyl-CoA product, reflecting their reduced Phs1 activity. Most of the mutant membranes tend to produce a reduced level of acyl-CoA, and a significant reduction was observed for the R83A, R141A, and G152A mutant membranes.

\subsection{The substrate specificity of Phs1 is affected by the E60A mutation}

To elucidate the effect of each mutation on the progression of the FA elongation cycle, we determined the chain-length profile of FA and 3-hydroxy-FA by reversed-phase TLC after conversion to the corresponding methyl esters (FA methyl ester (FAME) and 3-hydroxy-FAME, respectively) (Fig. 3A and B). The wild-type membrane elongated C16-CoA up to $\mathrm{C} 26-\mathrm{CoA}$ (C26 FAME in the figure), indicating that $\mathrm{C} 16-\mathrm{CoA}$ underwent 5 rounds of the elongation cycle (Fig. S1B). The upper two spots having the same Rf values (the ratio of distances migrated by the sample and the solvent front) with 3-hydroxy-C24-FAME and 3-hydroxy-C26-FAME should be associated only with C18-FAME and C20-FAME, respectively (Fig. 3A), since the wild-type membrane accumulated little 3-hydroxyacyl-CoA (Fig. 2A). The E60A, Q79A, E116A, and R119A mutant membranes also produced $\mathrm{C} 18$ - to $\mathrm{C} 26-\mathrm{CoAs}$, while the $\mathrm{C} 26-\mathrm{CoA}$ level was much lower than that produced by the wild-type membrane. As expected, these mutant membranes 
accumulated 3-hydroxyacyl-CoAs with C18, C20, and C22 (and probably C24 and C26).

The R83A, R141A, and G152A mutations had a more significant effect on the FA elongation cycle. Their acyl-CoA product and 3-hydroxyacyl-CoA intermediate were predominantly C18-CoA and 3-hydroxy-C18-CoA, respectively (Fig. 3A and B). Most of the FA elongation cycle in these mutant membranes seem to have stopped during and after the first round (Fig. S1B).

It is noteworthy that the E60A mutant membrane accumulated a rather high level of 3-hydroxy-C20- and 3-hydroxy-C22-CoA with reference to the level of 3-hydroxy-C18-CoA. Both levels were highest among the mutant membranes. It seems that the E60A mutant exhibits higher activity toward 3-hydroxy-C18-CoA than 3-hydroxy-C20- and 3-hydroxy-C22-CoA, whereas the wild-type Phs1 and other mutants maintain a similar activity toward 3-hydroxyacyl-CoA regardless of their chain-length. To test this possibility, we performed the FA elongation assay using acyl-CoA substrates with different chain-length (C16- to C24-CoAs) (Fig. S2A and B). The primary product of the wild-type membrane was acyl-CoA, irrespective of the substrate used. The 3-hydroxyacyl-CoA intermediate was detected less than $4 \%$ of the total product (acyl-CoA plus 3-hydroxyacyl-CoA). In contrast, in the E60A mutant membrane, the percentage of the acyl-CoA product decreased from $48 \%$ to $24 \%$ and to $<10 \%$ as the substrate chain-length increased from $\mathrm{C} 16$ to $\mathrm{C} 18$ and to $\geq \mathrm{C} 20$. For comparison, we subjected the R119A and R141A mutant membranes to the assay and found that the percentage of the acyl-CoA product was much less variable than the E60A mutant membrane. Thus, the lower activity toward 3-hydroxyacyl-CoA with longer chain-length seems characteristic of the E60A mutant. 


\subsection{Both $R 83 A$ and $G 152 A$ mutants display normal $K_{m}$ but reduced $V_{\max }$ values}

Three mutants (R83A, R141A, and G152A) exhibited severe FA elongation defects (Figs. 2 and 3). The Arg83 and Gly152 residues are located in the interior of the ER membrane, where the putative active site residues Tyr149 and Glu156 are present [8] (Fig. S3). Therefore, it is possible that the Arg83 and Gly152 residues are directly involved in the catalytic process, although the possible involvement of other residues cannot be excluded. On the other hand, the Arg141 residue is located outside the ER membrane (in the cytosolic loop), and the R141A mutant was sensitive to digitonin (Fig. 1C). Thus, the decreased activity of the R141A mutant is likely derived from a defect in structural integrity. To examine these possibilities, we determined the $K_{\mathrm{m}}$ and $V_{\max }$ values of the R83A, G152A, and R141A mutants and compared them with those of the wild-type Phs1 (Fig. 4A, B, and C). The $K_{\mathrm{m}}$ values of the R83A and G152A mutants were 2.70 and $2.50 \mu \mathrm{M}$, respectively, which were slightly lower than that of the wild-type Phs1 $(5.5 \mu \mathrm{M})$, suggesting that the substrate-enzyme affinity was not impaired by these mutations. The $V_{\max }$ values of the R83A and G152A mutants $(0.08$ and $0.11 \mathrm{pmol} / \mathrm{ng} / \mathrm{min}$, respectively) were lower by $\sim 1,000$-fold compared to that of the wild-type Phs1 (84.0 $\mathrm{pmol} / \mathrm{ng} / \mathrm{min})$. These results suggest that the catalytic process, but not the substrate recognition, was affected by the R83A and G152A mutations. The R141A mutant, however, exhibited a higher $K_{\mathrm{m}}$ and lower $V_{\max }$ than the wild type $\mathrm{Phs} 1\left(K_{\mathrm{m}}, 15.4 \mu \mathrm{M} ; V_{\max }\right.$, $0.54 \mathrm{pmol} / \mathrm{ng} / \mathrm{min}$ ) (Fig. 4C), which could imply that the structural defect had an adverse effect on both substrate recognition and catalytic process. 


\section{Discussion}

Phs1 and its homologs belong to a novel 3-hydroxyacyl-CoA dehydratase family with no sequence similarity to other protein families. The three-dimensional structure is not currently available for any of the Phs1 family members. We previously identified 9 amino-acid residues important for Phs1 function by expressing Phs1 mutants, in which each of the 22 conserved residues was replaced by Ala, in Tet-PHS1 cells [8]. Among these residues, the Tyr149 and Glu156 residues were found to be essential and considered as the putative active-site residues. However, the specific role of 7 other residues needs to be investigated. Accordingly, we have carried out several biochemical analyses on the corresponding Ala-substituted Phs1 mutants prepared previously.

The 3-hydroxyacyl-CoA dehydratase assay showed that the expected lower activity for all the mutants (Fig. 1A). Since the activity of the E60A, Q79A, and R141A mutants was reduced by treatment with the nonionic detergent digitonin (Fig. 1C), the Glu60, Gln79, and Arg141 residues seem important for the structural maintenance of Phs1. The immunoblot analysis revealed that the E60A protein level was the lowest (Fig. 1B). It is possible that the E60A mutant is destabilized in the ER membrane due to its reduced structural integrity.

From the results of the FA elongation assay (Fig. 3), we have classified the mutants into two classes (Class I: E60A, Q79A, E116A, and R119A; Class II: R83A, R141A, and G152A) (Fig. S1B). The Class I mutant membranes exhibited a moderate FA elongation defect and elongated $\mathrm{C} 16-\mathrm{CoA}$ to $\mathrm{C} 26-\mathrm{CoA}$ (the product of the $5^{\text {th }}$ elongation cycle), but in a lesser amount than the wild-type membrane. On the other hand, the Class II mutant membranes displayed a severe FA elongation defect yielding primarily $\mathrm{C} 18-\mathrm{CoA}$ (the product of the first elongation cycle) and 3-hydroxy-C18-CoA (the first round substrate for Phs1) in 11-22\% and 
$51-78 \%$ of the total products, respectively. Thus, most of the elongation cycle in the Class II mutant membranes ended during and after the first round. It appears that the Ala-substituted residues in the Class II mutants (Arg83, Arg141, and Gly152) are particularly important for the normal function of Phs1.

We previously predicted the membrane topology of Phs1 to have six membrane-spanning domains with both $\mathrm{N}$ - and C-termini facing the cytosol, and hypothesized the catalytic mechanism for Phs1 [8] in analogous to the proposed mechanism for the bacterial 3-hydroxyacyl-acyl carrier protein dehydratase FabA [12,13]. The Phs1-catalyzed $\alpha$, $\beta$-elimination of 3-hydroxyacyl-CoA may proceed through either a concerted or a stepwise manner involving enolization followed by dehydration. Accordingly, we have refined our originally proposed mechanism to illustrate these two possibilities (Fig. S4). Thus, during the catalytic process, the Tyr149 residue functions as a base in its phenoxide form abstracting the $\alpha$-hydrogen of the 3-hydroxyacyl-CoA substrate, and the Glu156 residue likely acts as an acid to donate a proton to the $\beta$-hydroxyl group of the substrate for its elimination as water. In the concerted mechanism, these two processes occur simultaneously in a concerted fashion, while the stepwise mechanism involves the initial formation of an enolate intermediate followed by elimination of the $\beta$-hydroxyl group. The Arg83 and Gly152 residues are probably important for the generation/stabilization of the Tyr149 phenoxide (by salt bridge formation) and the stabilization of the eliminated water as well as its removal from the active site (through hydrogen bond interaction). In addition, in the stepwise mechanism, the Gly152 residue could stabilize the enolate intermediate by hydrogen bonding to the enolate oxygen. Based on the report that yeast ER membrane proteins typically traverse the membrane via the hydrophobic stretch of 15 amino-acid residues [14], as well as the hydropathy plot of Phs1 
[15], we have slightly modified our original membrane topology model for Phs1 by revising the length of the transmembrane domain from 18 to 15 residues (Fig. S3). In the revised model, the 2 catalytic residues (Tyr149 and Glu156), as well as the 2 key residues (Arg83 and Gly152), still remain in the interior of the ER membrane. The proposed direct involvement of the Arg83 and Gly152 residues in the catalytic process is consistent with the enzyme kinetics data revealing that both $\mathrm{R} 83 \mathrm{~A}$ and G152A mutations resulted in the Phs1 mutants with the greatly reduced $V_{\max }$ values without affecting their $K_{\mathrm{m}}$ values (Fig. 4B). On the other hand, the Arg141 residue resides in the cytoplasmic domain of Phs1 (Fig. S3), and hence its direct involvement in the catalytic process is unlikely. Rather, the Arg141 residue may be required for structural maintenance of the active site, since the activity of the R141A mutant was decreased upon digitonin treatment (Fig. 1C). Indeed, the R141A mutation had a negative effect on both $K_{\mathrm{m}}$ and $V_{\max }$ values (Fig. 4C).

The E60A mutant seemed to have the highest substrate specificity to 3-hydroxy-C18-CoA over the other longer-chain 3-hydroxyacyl-CoAs (Figs. 3 and S2). For example, the ratio of 3-hydroxy-C20-CoA to 3-hydroxy-C18-CoA was $87 \%$ in E60A mutant membrane, whereas the ratio was much lower in other Class I and Class II mutant membranes (47-52\% and 6-32\%, respectively) (Fig. 3B). This finding is interesting, considering that the C16- and C18-FAs are the major FA components of glycerophospholipids, the most abundant lipids in the ER. During the FA elongation cycle, an acyl-CoA substrate is believed to be located in the cytoplasmic leaflet of the ER lipid bilayer with its CoA moiety in the cytosol. Through repetitive addition of $\mathrm{C} 2$ units, the methyl end of the acyl chain traverses from the cytosolic to the luminal leaflet. The Glu60 residue is located near the interface between the two leaflets of the ER lipid bilayer (Fig. S3). Accordingly, we speculate that the E60A mutation causes a 
structural change that interferes with the accommodation of longer FA chains, especially longer than C20.

In the present study, we have obtained biochemical information revealing the amino-acid residues important for the catalysis, structural maintenance, and substrate specificity of Phs1. Since being conserved among the Phs1 family, these identified residues may play a similar role in the eukaryotic HACD proteins. The mutation in dog HACD1 is found to cause canine centronuclear myopathy [16]. Future studies may identify additional HACD-related disorders. Therefore, our findings will be useful for understanding the relationships among mutations, enzyme activities, and pathologies. 
Acknowledgements: This work was supported by a Grant-in-Aid for Scientific Research (B) (23370057) from the Japan Society for the Promotion of Science (JSPS). We are grateful to Dr. K. Obara for helpful discussion and to Dr. T. Toyokuni for scientific editing of the manuscript. 


\section{References}

[1] Kihara, A. (2012) Very long-chain fatty acids: elongation, physiology and related disorders. J. Biochem. 152, 387-395.

[2] Revardel, E., Bonneau, M., Durrens, P. and Aigle, M. (1995) Characterization of a new gene family developing pleiotropic phenotypes upon mutation in Saccharomyces cerevisiae. Biochim. Biophys. Acta 1263, 261-265.

[3] Rantakari, P., Lagerbohm, H., Kaimainen, M., Suomela, J.P., Strauss, L., Sainio, K., Pakarinen, P. and Poutanen, M. (2010) Hydroxysteroid (17 $\beta$ ) dehydrogenase 12 is essential for mouse organogenesis and embryonic survival. Endocrinology 151, 1893-1901.

[4] Nugteren, D.H. (1965) The enzymic chain elongation of fatty acids by rat-liver microsomes. Biochim. Biophys. Acta 106, 280-290.

[5] Denic, V. and Weissman, J.S. (2007) A molecular caliper mechanism for determining very long-chain fatty acid length. Cell 130, 663-677.

[6] Ikeda, M., Kanao, Y., Yamanaka, M., Sakuraba, H., Mizutani, Y., Igarashi, Y. and Kihara, A. (2008) Characterization of four mammalian 3-hydroxyacyl-CoA dehydratases involved in very long-chain fatty acid synthesis. FEBS Lett. 582, $2435-2440$.

[7] Kihara, A., Mitsutake, S., Mizutani, Y. and Igarashi, Y. (2007) Metabolism and biological functions of two phosphorylated sphingolipids, sphingosine 1-phosphate and ceramide 1-phosphate. Prog. Lipid Res. 46, 126-144.

[8] Kihara, A., Sakuraba, H., Ikeda, M., Denpoh, A. and Igarashi, Y. (2008) Membrane topology and essential amino acid residues of Phs1, a 3-hydroxyacyl-CoA 
dehydratase involved in very long-chain fatty acid elongation. J. Biol. Chem. 283, 11199-11209.

[9] Brachmann, C.B., Davies, A., Cost, G.J., Caputo, E., Li, J., Hieter, P. and Boeke, J.D. (1998) Designer deletion strains derived from Saccharomyces cerevisiae S288C: a useful set of strains and plasmids for PCR-mediated gene disruption and other applications. Yeast 14, 115-132.

[10] Uemura, S., Kihara, A., Iwaki, S., Inokuchi, J. and Igarashi, Y. (2007) Regulation of the transport and protein levels of the inositol phosphorylceramide mannosyltransferases Csg1 and Csh1 by the $\mathrm{Ca}^{2+}$-binding protein Csg2. J. Biol. Chem. $282,8613-8621$.

[11] Ohno, Y., Suto, S., Yamanaka, M., Mizutani, Y., Mitsutake, S., Igarashi, Y., Sassa, T. and Kihara, A. (2010) ELOVL1 production of C24 acyl-CoAs is linked to C24 sphingolipid synthesis. Proc. Natl. Acad. Sci. USA 107, 18439-18444.

[12] Leesong, M., Henderson, B.S., Gillig, J.R., Schwab, J.M. and Smith, J.L. (1996) Structure of a dehydratase-isomerase from the bacterial pathway for biosynthesis of unsaturated fatty acids: two catalytic activities in one active site. Structure 4, 253-264.

[13] White, S.W., Zheng, J., Zhang, Y.M. and Rock, C.O. (2005) The structural biology of type II fatty acid biosynthesis. Annu. Rev. Biochem. 74, 791-831.

[14] Levine, T.P., Wiggins, C.A. and Munro, S. (2000) Inositol phosphorylceramide synthase is located in the Golgi apparatus of Saccharomyces cerevisiae. Mol. Biol. Cell 11, 2267-2281.

[15] Kyte, J. and Doolittle, R.F. (1982) A simple method for displaying the hydropathic character of a protein. J. Mol. Biol. 157, 105-132. 
[16] Pelé, M., Tiret, L., Kessler, J.L., Blot, S. and Panthier, J.J. (2005) SINE exonic insertion in the PTPLA gene leads to multiple splicing defects and segregates with the autosomal recessive centronuclear myopathy in dogs. Hum. Mol. Genet. 14, $1417-1427$. 


\section{Figure legends}

Fig. 1. The Glu60, Gln79, Arg83, Glu116, Arg119, Arg141, and Gly152 residues are important for Phs1 activity. (A-C) Total membrane fractions were prepared from YTY19 (wild type; WT), YTY20 (E60A), YTY21 (Q79A), YTY22 (R83A), YTY23 (E116A), YTY24 (R119A), YTY25 (R141A), and YTY26 (G152A) cells. (A) Total membrane fractions $(4 \mu \mathrm{g})$ were incubated with $3.6 \mu \mathrm{M}$ 3-hydroxy $\left[1-{ }^{14} \mathrm{C}\right]$ palmitoyl-CoA $(0.01 \mu \mathrm{Ci})$ for 15 min at $37{ }^{\circ} \mathrm{C}$. After termination of the reactions, lipids were saponified, acidified, extracted, and separated by normal-phase TLC. The radioactivity associated with the reaction product 2,3-trans hexadecenoic acid was quantified using a bioimaging analyzer BAS-2500 (Fuji Film, Tokyo, Japan). Values represent the mean \pm S.D. of three independent experiments. Statistically significant differences from the wild type are indicated $\left({ }^{*} \mathrm{p}<0.05\right.$, ** $\mathrm{p}<0.01$; $\mathrm{t}$-test). (B) Total membrane fractions $(3 \mu \mathrm{g})$ were separated by SDS-PAGE and detected by immunoblotting with anti-FLAG antibody or, to normalize protein loading, with anti-Pma1 (a plasma membrane protein) antibody. The values below the gel image are the relative amount of each Phs1-3xFLAG, after normalization with Pma1, to the wild-type protein. (C) Total membrane fractions $(4 \mu \mathrm{g})$ were subjected to a 3-hydroxyacyl-CoA dehydratase assay as in (A) with or without $1 \%$ digitonin. Values represent the mean \pm S.D. of three independent experiments. Statistically significant differences are indicated $\left({ }^{*} \mathrm{p}<0.05\right.$; t-test).

Fig. 2. The R83A, R141A, G152A mutant membranes exhibit a decreased acyl-CoA production in the FA elongation cycle. (A and B) Total membrane fractions ( $20 \mu \mathrm{g})$ prepared from YTY19 (wild type; WT), YTY20 (E60A), YTY21 (Q79A), YTY22 (R83A), YTY23 
(E116A), YTY24 (R119A), YTY25 (R141A), and YTY26 (G152A) cells were incubated with $50 \mu \mathrm{M}$ palmitoyl-CoA and $27.3 \mu \mathrm{M}\left[2-{ }^{14} \mathrm{C}\right]$ malonyl-CoA $(0.075 \mu \mathrm{Ci})$ for $30 \mathrm{~min}$ at 37 ${ }^{\circ} \mathrm{C}$. Lipids were saponified, acidified, extracted, and separated by normal-phase TLC. Using a bioimaging analyzer BAS-2500, the radioactivity was detected (A) and quantified (B). Values represent the mean \pm S.D. of three independent experiments. Statistically significant differences from the wild type are indicated $(* \mathrm{p}<0.05, * * \mathrm{p}<0.01 ; \mathrm{t}$-test $)$.

Fig. 3. Substrate specificity is affected in the E60A mutant. (A and B) Total membrane fractions $(20 \mu \mathrm{g})$ prepared from YTY19 (wild type; WT), YTY20 (E60A), YTY21 (Q79A), YTY22 (R83A), YTY23 (E116A), YTY24 (R119A), YTY25 (R141A), and YTY26 (G152A) cells were incubated with $20 \mu \mathrm{M}$ palmitoyl-CoA, $73 \mu \mathrm{M}$ malonyl-CoA, and $27.3 \mu \mathrm{M}$ $\left[2-{ }^{14} \mathrm{C}\right]$ malonyl-CoA $(0.075 \mu \mathrm{Ci})$ for $30 \mathrm{~min}$ at $37{ }^{\circ} \mathrm{C}$. Lipids were saponified, acidified, and extracted. After methyl esterification, the esters were separated by reversed-phase TLC. Using a bioimaging analyzer BAS-2500, the radioactivity was detected (A) and quantified (B). Values represent the mean \pm S.D. of three independent experiments. For the wild type, the radioactivity of the two spots corresponding to C18-FAME/3-hydroxy-C24 FAME and C20-FAME/3-hydroxy-C26-FAME was considered to be derived mainly from C18-FAME and C20-FAME, respectively, since little accumulation of 3-hydroxy-CoA (i.e., 3-hydroxy-FAME) (<10\%) was detected in the wild-type membrane (See Fig. 2A).

Fig. 4. The R83A and G152A mutations result in a reduced $V_{\max }$ value. (A) Total membrane fractions $(0.16 \mu \mathrm{g})$ prepared from YTY19 (wild type; WT) were incubated with 2, 4, 9, 12, 16, 24, and $36 \mu \mathrm{M}$ 3-hydroxy $\left[1-{ }^{14} \mathrm{C}\right]$ palmitoyl-CoA $(0.1 \mu \mathrm{Ci} / \mu \mathrm{l})$ for $1 \mathrm{~min}$ at $37{ }^{\circ} \mathrm{C}$. After 
termination of the reaction, lipids were saponified, acidified, extracted, and separated by normal-phase TLC. The radioactivity associated with the reaction product 2,3-trans hexadecenoic acid was quantified using a bioimaging analyzer BAS-2500. The Phs1-3xFLAG level in the membrane fractions used was determined relative to the level of the 3xFLAG-maltose binding protein standard, and used for the calculation of the specific activity. The obtained values were expressed in a Lineweaver-Burk plot with a linear regression line. $\mathrm{R}$ is a correlation coefficient. (B) Total membrane proteins (4 $\mu \mathrm{g}$ ) prepared from YTY22 (R83A) and YTY26 (G152A) cells were incubated with 2, 4, 8, and $16 \mu \mathrm{M}$ 3-hydroxy $\left[{ }^{14} \mathrm{C}\right]$ palmitoyl-CoA for $15 \mathrm{~min}$ at $37{ }^{\circ} \mathrm{C}$. The $K_{\mathrm{m}}$ and $V_{\max }$ values were determined as in (A). (C) Total membrane proteins $(4 \mu \mathrm{g})$ prepared from YTY25 (R141A) cells were incubated with $0.5,4,8$, and $16 \mu \mathrm{M}$ 3-hydroxy $\left[{ }^{14} \mathrm{C}\right]$ palmitoyl-CoA for $15 \min$ at $37{ }^{\circ} \mathrm{C}$. The $K_{\mathrm{m}}$ and $V_{\max }$ values were determined as in (A). 
Figure 1

A

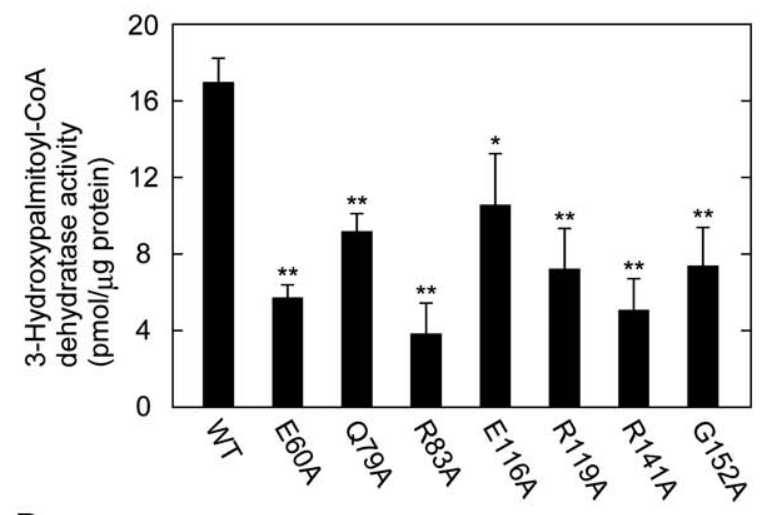

B
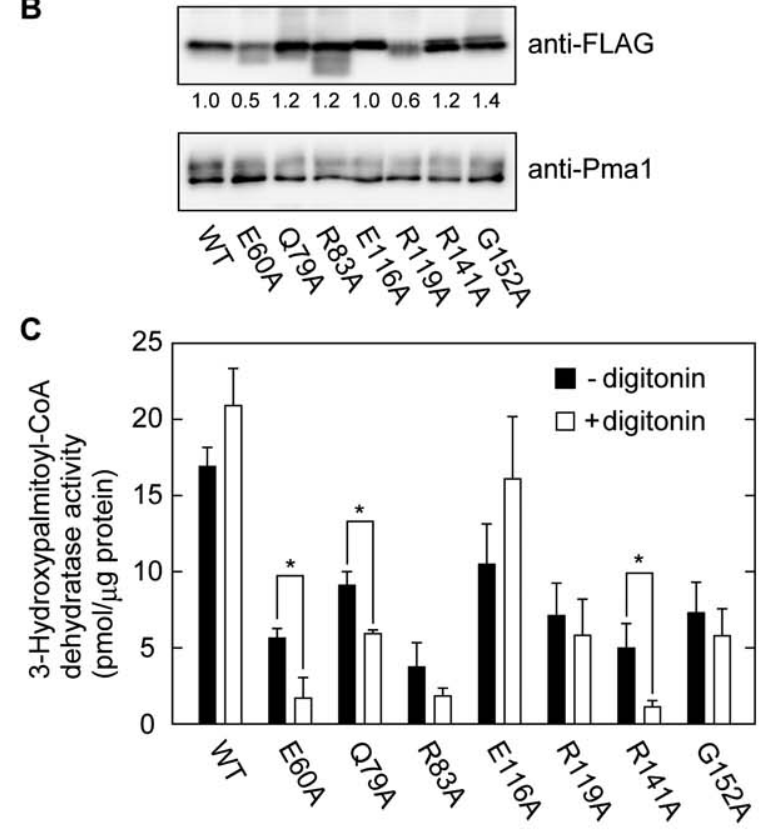
Figure 2

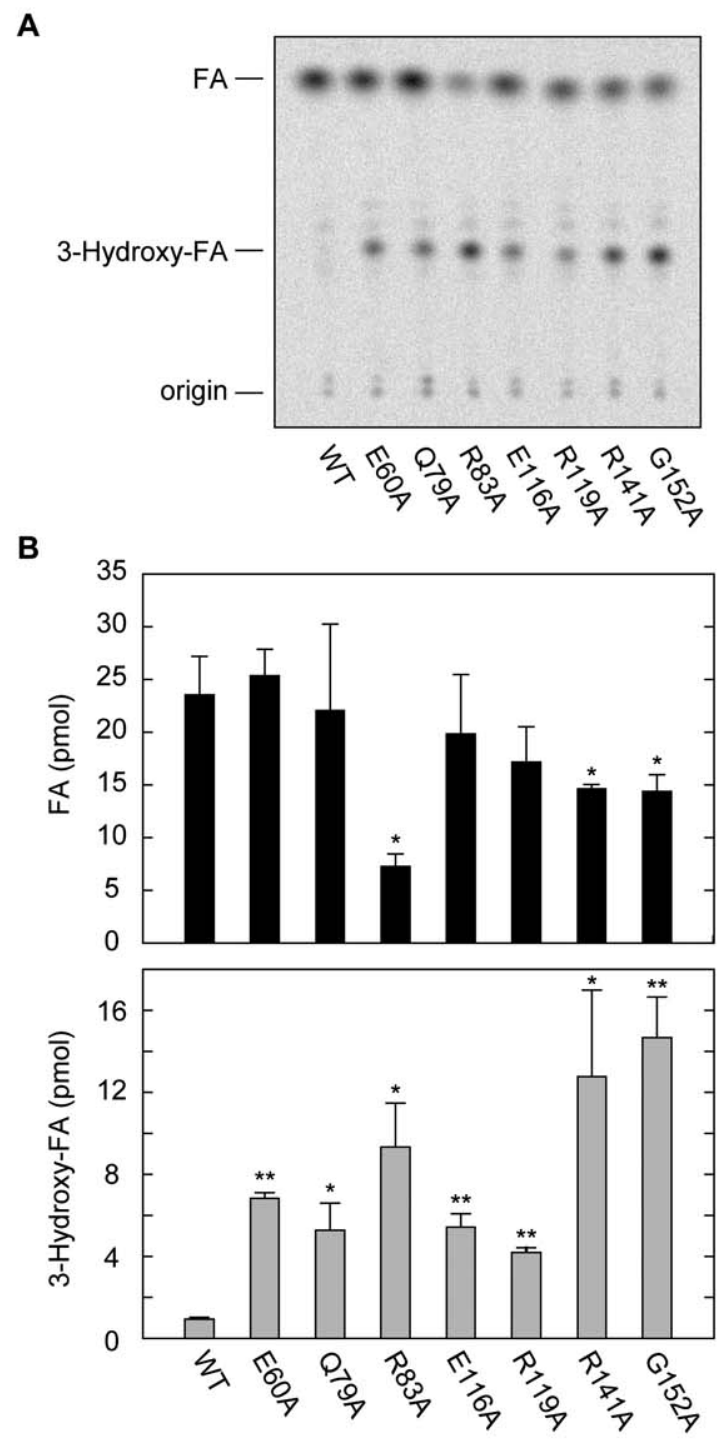


Figure 3

A
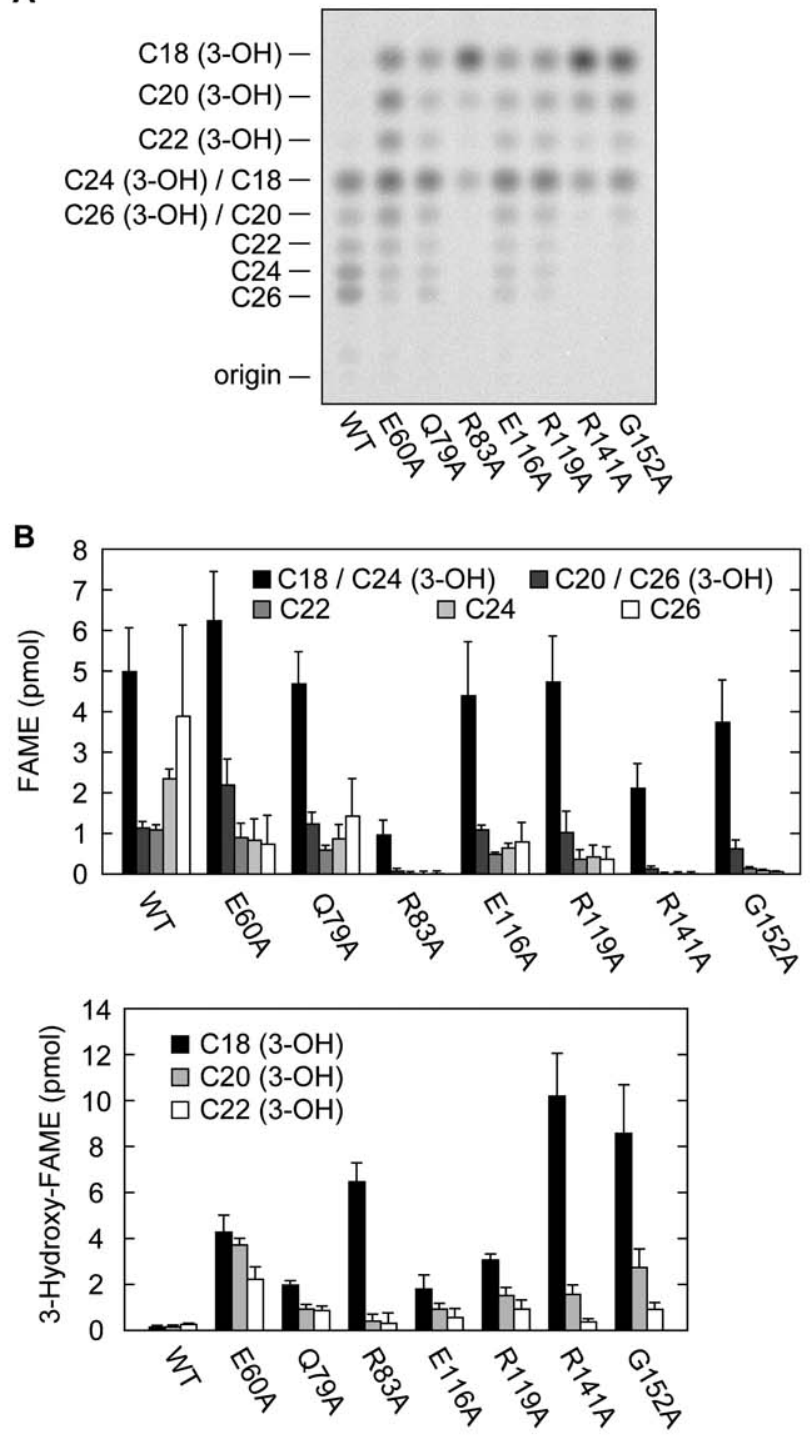


\section{Figure 4}

A WT: $K_{\mathrm{m}}, 5.5 \mu \mathrm{M} ; V_{\max }, 84.0 \mathrm{pmol} / \mathrm{ng} / \mathrm{min}$

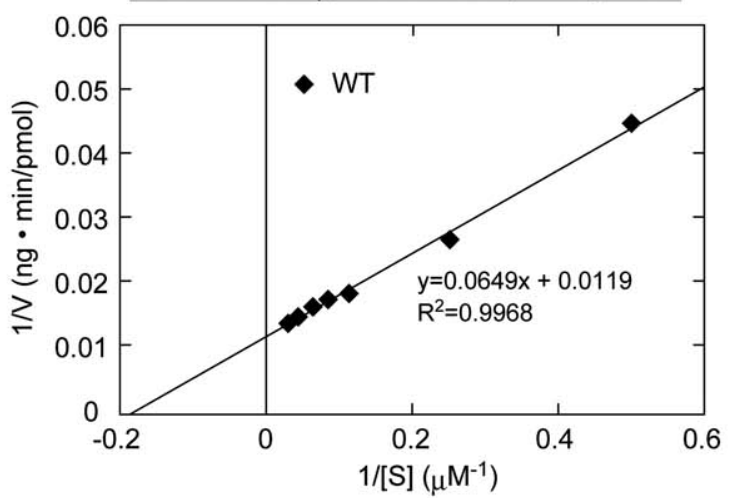

B

R83A: $K_{m}, 2.70 \mu \mathrm{M} ; V_{\max }, 0.08 \mathrm{pmol} / \mathrm{ng} / \mathrm{min}$

G152A: $K_{\mathrm{m}}, 2.50 \mu \mathrm{M} ; V_{\max }, 0.11 \mathrm{pmol} / \mathrm{ng} / \mathrm{min}$

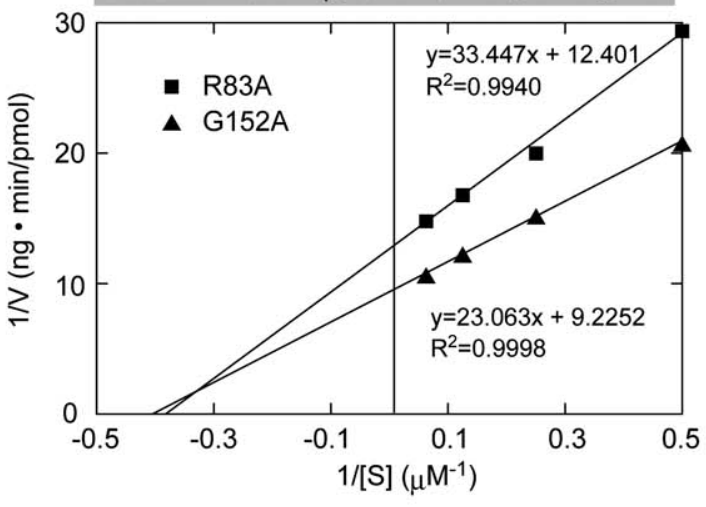

C

R141A: $K_{m}, 15.4 \mu \mathrm{M} ; V_{\max }, 0.54 \mathrm{pmol} / \mathrm{ng} / \mathrm{min}$

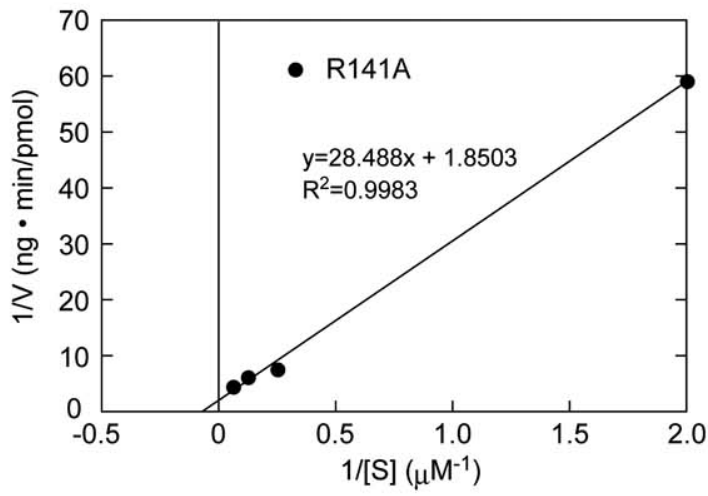

